To the Editors:

\title{
Periodic changes in behaviour in a child with pseudohypoparathyroidism
}

We present here a preliminary communication about a child with pseudohypoparathyroidism (PHP) and its neuropsychiatric manifestations. The case illustrates an unusual presentation, and a diagnostic and management dilemma. PHP comprises a heterogeneous group of genetically determined disorders where the clinical features are due to end organ resistance to parathyroid hormone.

A 9-year old prepubertal girl, gave a history of periodic changes in behaviour of 16 months' duration. The parents described the changes in behaviour as follows. She becomes less communicative and less interactive with

Vol. 46, No. 2, June 2001 others and clinging to the mother. Vomiting or retching, poor food intake, disturbed sleep pattern, and sometimes feelings of fearfulness were other features. She was unable to give a descriptive account of her fears but referred to something or someone frightening her. Presence of abnormal visual or auditory perceptual experiences could not be established. She refused to go to school, play or engage in any of her usual recreational activities during these episodes. The periodic changes were regular with each episode lasting 10 to 11 days and recurring at 13 to 14 day intervals. At the two ends of each episode, the change of behaviour was usually confined to only part of the day. 
14 months after the onset of symptoms, she developed tetany with carpopedal spasms and a positive Chvostek's sign. Laboratory investigations showed a serum calcium of $1.56 \mathrm{mmol} /$ (normal 2.02 to $2.6 \mathrm{mmol} / \mathrm{h}$ ) and serum phosphate of $4.4 \mathrm{mg} / \mathrm{dl}$ (normal 2.68 to $4.5 \mathrm{mg} / \mathrm{dl}$ ). $24-$ hour urinary calcium excretion was $72 \mathrm{mg}$ (normal 100 to 300 $\mathrm{mg}$ ) and phosphate $0.35 \mathrm{mg}$ (normal 0.3 to $1 \mathrm{mg}$ ). Serum parathyroid hormone level was $1338 \mathrm{micrograml} / \mathrm{ml}$ (normal 12 to $72 \mathrm{microgram} / \mathrm{ml}$ ). Other relevant investigations such as serum albumin, serum alkaline phosphatase, serum sodium and potassium were all within normal range. EEG showed generalised bursts of delta waves with no lateralising signs, suggestive of a metabolic encephalopathy. CT brain scan was normal.

She had been taking calcium supplements, vitamin D and alfacalcidol but not long enough to produce any significant changes in the serum calcium level. She is also taking fluoxetine. The outcome of the treatment is not available at the present time.

Neuropsychiatric manifestations are known to occur in PHP. The possible aetiology of this child's behaviour change became apparent only after she developed neuromuscular manifestations of hypocalcaemia. Onset of these was much later during the course of illness compared to behavioural and emotional changes. The other unusual feature in this case is the cyclical recurrence of symptoms, which is difficult to explain. Also, the combination of symptoms of vomiting, social withdrawal and fearfulness has not been previously documented in association with PHP.

Factors precipitating overt symptoms in subclinical PHP are not known but vitamin D deficiency might be a factor (1). Most published accounts of such manifestations are limited to anecdotal reports in adults and adolescents. Known presentations are psychotic states, anxiety disorders and intellectual retardation. Rapid cycling bipolar affective illness has been reported where symptoms disappeared when the serum calcium level was corrected (1). In other cases, the psychiatric symptoms needed neuroleptic medication in addition (2). A periodic psychotic state has also been described (3). Mental retardation is known to occur with Type 1 PHP (4). Other psychiatric symptoms such as delirium, poor concentration, emotional lability, impaired intellectual function also have been described in PHP (5).

\section{References}

1. Pollard AJ, Prendergast M, al-Hammouri F, Rayner PH, Shaw NJ. Different subtypes of pseudohypoparathyroidism in the same family with an unusual psychiatric presentation of the index case. Archives of Diseases of Childhood 1994; 70: 99102.

2. Preskom SH, Reveley A. Pseudohypoparathyroidism and Capgra's syndrome. British Joumal of Psychiatry 1978; 133: 34-7.

3. Furukawa T. Periodic psychosis associated with pseudopseudohypoparathyroidism. Joumal of Nervous and Mental Disorders 1991; 179: 637-8.

4. Farfel Z, Freidman E. Mental deficiency in pseudohypoparathyroidism type I is associated with Ns-protein deficiency. Annals of Internal Medicine 1986; 105: 197-9.

S. Denko JD, Kaelbling R. The psychiatric aspects of hypoparathyroidism. Acta Psychiatrica Scandinavica 1962; 164: 1. 70.

H Perera, Senior Lecturer, Department of Psychological Medicine, University of Colombo, J C Wijesekara, Neurologist, National Hospital of Sri Lanka, and U Hewage, Senior Lecturer in Medicine, Faculty of Medical Sciences, University of Sri Jayawardenepura. 